\title{
Existence and Nonexistence of Global Solutions for Nonlinear Parabolic Equations
}

By

\author{
Masayoshi Tsutsumi*
}

\section{Introduction}

Let $\Omega$ be a bounded domain in $R^{n}$ with smooth boundary $\partial \Omega, p$ a real number $\geqq 2$ and $\alpha$ a nonnegative real number. In this paper we consider the initial-boundary value problems of the form

$$
\begin{gathered}
\frac{\partial u}{\partial t}=\sum_{i=1}^{n} \frac{\partial}{\partial x_{i}}\left(\left|\frac{\partial u}{\partial x_{i}}\right|^{p-2} \frac{\partial u}{\partial x_{i}}\right)+u^{1+\alpha}, t>0, x \in \Omega, \\
u(x, 0)=u_{0}(x), \quad x \in \Omega, \\
u(x, t)=0, \quad x \in \partial \Omega, \quad t \geqq 0 .
\end{gathered}
$$

In a recent work [1], Fujita gave existence and nonexistence theorems for global solutions of the equation

$$
\frac{\partial u}{\partial t}=\Delta u+u^{1+\alpha}, t>0, x \in \Omega,
$$

with conditions (1.2), (1.3).

In this paper our purpose is to obtain analogous results for the problem (1.1)-(1.3). ${ }^{1)}$ Roughly speaking, our results are as follows:

1) if $p>2+\alpha$, the problem (1.1)-(1.3) has global (nonnegative) solutions whenever initial functions $u_{0}(x)$ (are nonnegative and) belong to some Sobolev space.

Received May 8, 1972.

Communicated by S. Matsuura.

* Department of Applied Physics, Waseda University., Tokyo, Japan.

1) This problem was proposed by Lions in his book [2]. 
$2)$ if $p<2+\alpha$, for sufficiently small (nonnegative) initial function $u_{0}(x)$, the problem (1.1)-(1.3) has a global (nonnegative) solution. If $u_{0}(x)$ is nonnegative and large enough, the solution blows up in a finite time.

We shall solve the problem (1.1)-(1.3) by considering the "truncated" equation

$$
\frac{\partial u}{\partial t}=\sum_{i=1}^{n} \frac{\partial}{\partial x_{i}}\left(\left|\frac{\partial u}{\partial x_{i}}\right|^{p-2} \frac{\partial u}{\partial x_{i}}\right)+\varphi(u), t>0, x \in \Omega,
$$

where $\varphi(u)=\left\{0\right.$ if $u<0, u^{1+\alpha}$ if $\left.u \geqq 0\right\}$, with conditions (1.2) and (1.3), and proving the maximum principle for the weak solution of the equation $\left(1.1^{\prime}\right)$.

Below, $\S 2$ is devoted to preliminaries. Global existence and uniqueness theorems of the case $p>2+\alpha$ and $p<2+\alpha$ are stated in $\S 3$ and $\S 4$, respectively. In $\S 5$ blowing up of solutions is discussed. In the final section we consider the equation

$$
\frac{\partial u}{\partial t}=\sum_{i=1}^{n} \frac{\partial}{\partial x_{i}}\left(\left|\frac{\partial u}{\partial x_{i}}\right|^{p-2} \frac{\partial u}{\partial x_{i}}\right)-u^{1+\alpha}, t>0, x \in \Omega,
$$

with conditions (1.2) and (1.3).

\section{Preliminaries}

We shall use the notations employed in the book of Lions [2].

The following lemmas are well known. The reader is referred to Ladyzhenskaya, Solonnikov and Uralceva [3] for proofs.

Lemma 1. For any function $u(x) \in W_{0}^{1, p}(\Omega), p \geqq 1$ and $r \geqq 1$, the inequality

$$
\|u\|_{L^{q}(\Omega)} \leqq C_{1}\left(\sum_{i=1}^{n}\left\|\frac{\partial u}{\partial x_{i}}\right\|_{L^{p}(\Omega)}\right)^{\rho}\|u\|_{L^{r}(\Omega)}^{\bar{r}_{(}^{\rho}}
$$

is valid, where

$$
\rho=\left(\frac{1}{r}-\frac{1}{q}\right)\left(\frac{1}{n}-\frac{1}{p}+\frac{1}{r}\right)^{-1}
$$


and:

1) for $p \geqq n=1, \quad r \leqq q \leqq \infty$;

2) for $n>1$ and $p<n, \quad r \leqq q \leqq n p /(n-p)$ if $r \leqq n p /(n-p)$ and $n p /(n-p) \leqq q \leqq r$ if $r \geqq n p /(n-p)$;

3) for $p=n>1, \quad r \leqq q<\infty$;

4) for $p>n>1, \quad r \leqq q \leqq \infty$.

The constant $C_{1}$ depends only on $n, p, q$ and $r$.

Lemma 2. For any function $u(x) \in W_{0}^{1, p}(\Omega)$, we have

$$
\|u\|_{L^{q}(\Omega)} \leqq C_{2}\left(\sum_{i=1}^{n}\left\|\frac{\partial u}{\partial x_{i}}\right\|_{L^{p}(\Omega)}^{p}\right)^{1 / p}
$$

where $1 \leqq q \leqq n p /(n-p)$ if $n>p$ and $1 \leqq q<\infty$ if $n \leqq p$. The constant $C_{2}$ depends only on $\Omega, n, p$ and $q$. If $n<p$, the functions in $W_{0}^{1, p}(\Omega)$ are continuous and

$$
\sup |u| \leqq C_{3}\left(\sum_{i=1}^{n}\left\|\frac{\partial u}{\partial x_{i}}\right\|_{L^{p}(\Omega)}^{p}\right)^{1 / p}
$$

where $C_{3}$ depends only on $\Omega, n$ and $p$.

Next lemmas will be used in $\S 4$.

Lemma 3. Suppose that $u \in W_{0}^{1, p}(\Omega)$ where $p<2+\alpha$ if $n \leqq p$ and $p<2+\alpha \leqq n p /(n-p)$ if $n>p . \quad$ Put

$$
J(u)=\frac{1}{p} a(u)-\frac{1}{2+\alpha} b(u)
$$

where

$$
a(u)=\sum_{i=1}^{n}\left\|\frac{\partial u}{\partial x_{i}}\right\|_{L^{p}(\Omega)}^{p}, \quad b(u)=\int_{\Omega} \Phi(u(x)) d x
$$

in which $\Phi(u)=\left\{0\right.$ if $u<0, u^{2+\alpha}$ if $\left.u \geqq 0\right\}$.

Then we have

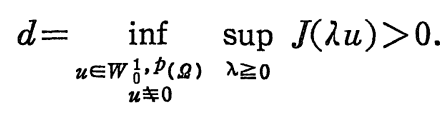


Proof. Evidently we get

$$
J(\lambda u)=\frac{\lambda^{p}}{p} a(u)-\frac{\lambda^{2+\alpha}}{2+\alpha} b(u) .
$$

Lemma 2 yields

$$
|b(u)| \leqq C_{2}^{2+\alpha} a(u)^{(2+\alpha) / p}
$$

Hence

$$
\begin{aligned}
\sup _{\lambda \geqq 0} J(\lambda u) & =J\left(\left(\frac{a(u)}{b(u)}\right)^{1 /(2+\alpha-p)} u\right) \\
& =\frac{2+\alpha-p}{(2+\alpha) p}\left(\frac{a(u)^{2+\alpha}}{b(u)^{p}}\right)^{1 /(2+\alpha-p)} \\
& \geqq \frac{2+\alpha-p}{(2+\alpha) p}\left(\frac{1}{C_{2}}\right)^{(2+\alpha) p /(2+\alpha-p)}>0
\end{aligned}
$$

Q.E.D.

We then introduce the stable set $\mathscr{W}$ (see Sattinger [4], Lions [2]):

$$
\mathscr{W}=\left\{u \mid u \in W_{0}^{1, p}(\Omega), 0 \leqq J(\lambda u)<d, \lambda \in[0,1]\right\} .
$$

Lemma 4. We have

$$
\mathscr{W}=\mathscr{W}_{*} \cup\{0\}
$$

where

$$
\mathscr{W}_{*}=\left\{u \mid u \in W_{0}^{1, p}(\Omega), a(u)-b(u)>0, J(u)<d\right\}
$$

Proof. 1) Suppose that $u \in \mathscr{W}, u \neq 0$. Then we have

$$
\sup _{\lambda \geqq 0} J(\lambda u)=J\left(\left(\frac{a(u)}{b(u)}\right)^{1 /(2+\alpha-p)} u\right) \geqq d
$$

and hence

$$
\left(\frac{a(u)}{b(u)}\right)^{1 /(2+\alpha-p)}>1
$$

which implies $u \in \mathscr{W}_{*}$.

2) Reciprocally, let $u \in \mathscr{W}_{*}$. Then we have 


$$
\sup _{\lambda \in[0,1]} J(\lambda u)=J(u)<d
$$

since $\frac{\partial}{\partial \lambda} J(\lambda u)>0$ for $0<\lambda \leqq 1$ and $\left.J(\lambda u)\right|_{\lambda=0}=0$.

Q.E.D.

Remark 1. Sattinger [4] introduced the stable set (potential well) in order to prove the global existence of solutions for semilinear hyperbolic equations which have not necessarily positive definite energy. We shall show below that analogous method is also applicable for the problem (1.1)(1.3) when $p<2+\alpha$.

Remark 2. The constant $d$ may be infinite. Sattinger supposed and used the finiteness of $d$ in his proofs. However our method does not require the finiteness of $d$.

The following lemma concerns the finiteness of $d$.

Lemma 5. Consider the following nonlinear positive eigenvalue problem:

$$
\begin{gathered}
\sum_{i=1}^{n} \frac{\partial}{\partial x_{i}}\left(\left|\frac{\partial u}{\partial x_{i}}\right|^{p-2} \frac{\partial u}{\partial x_{i}}\right)+\lambda \varphi(u)=0, \quad \lambda>0 \\
\left.u\right|_{\partial \Omega}=0,
\end{gathered}
$$

where $p<2+\alpha$ if $n \leqq p$ and $p<2+\alpha<n p /(n-p)$ if $n>p$.

If the problem (2.8) has an eigenfunction $u_{\lambda}$ for some $\lambda>0$, then the constant $d$ is finite and the stable set $\mathscr{W}$ is bounded in $W_{0}^{1, p}(\Omega)$.

Proof. We have

$$
J\left(\left(\frac{a\left(u_{\lambda}\right)}{b\left(u_{\lambda}\right)}\right)^{1 /(2+\alpha-p)} u_{\lambda}\right) \geqq d
$$

On the other hand, from (2.8), we get

$$
a\left(u_{\lambda}\right)-\lambda b\left(u_{\lambda}\right)=0 .
$$

Hence 


$$
\begin{aligned}
J\left(\left(\frac{a\left(u_{\lambda}\right)}{b\left(u_{\lambda}\right)}\right)^{1 /(2+\alpha-p)} u_{\lambda}\right) & =J\left(\lambda^{1 /(2+\alpha-p)} u_{\lambda}\right) \\
& =\frac{2+\alpha-p}{(2+\alpha) p} \cdot a\left(u_{\lambda}\right) \cdot \lambda^{p /(2+\alpha-p)}
\end{aligned}
$$

from which it follows that $d$ is finite.

Let $u \in \mathscr{W}$ and $u \neq 0$. Then from Lemma 4 , we have

$$
a(u)-b(u)>0 \text {. }
$$

Hence

$$
\begin{aligned}
d>J(u) & =\frac{1}{p} a(u)-\frac{1}{2+\alpha} b(u) \\
& \geqq \frac{2+\alpha-p}{(2+\alpha) p} a(u)
\end{aligned}
$$

which implies

$$
a(u) \leqq \frac{(2+\alpha) p}{2+\alpha-p} d
$$

Thus the stable set $\mathscr{W}$ is contained in the sphere:

$$
\left\{v \mid v \in W_{0}^{1, p}(\Omega), a(v) \leqq \frac{(2+\alpha) p}{2+\alpha-p} d\right\}
$$

\section{Global Existence and Uniqueness When $p>2+\alpha$}

Theorem 1. Suppose that $u_{0}(x) \in W_{0}^{1, p}(\Omega), p>2+\alpha$. Then there exists a function $u(x, t)$ such that

$$
\begin{gathered}
u \in L^{\infty}\left(0, T ; W_{0}^{1, p}(\Omega)\right), \\
\partial u / \partial t \in L^{2}\left(0, T ; L^{2}(\Omega)\right)
\end{gathered}
$$

and which satisfies $\left(1.1^{\prime}\right)$ in a generalized sense.

If $n<p$, the function $u(x, t)$ is uniquely determined by the initial function $u_{0}(x)$.

If $u_{0}(x) \geqq 0$ a.e. in $\Omega$, the function $u(x, t) \geqq 0$ a.e. in $\Omega$ for any fixed $t \geqq 0$ and is a solution of the problem (1.1)-(1.3). 
Remark 3. After eventual modification on a set of measure zero $u$ is continuous from $[0, T] \rightarrow L^{2}(\Omega)$.

Proof of Theorem 1. Put

$$
A v=-\sum_{i=1}^{n} \frac{\partial}{\partial x_{i}}\left(\left|\frac{\partial v}{\partial x_{i}}\right|^{p-2} \frac{\partial v}{\partial x_{i}}\right)
$$

We easily see that $A$ is a strictly monotone hemicontinuous bounded and coercive operator from $W_{0}^{1, p}(\Omega) \rightarrow W^{-1, p /(p-1)}(\Omega)$.

We shall employ the Galerkin's method. Let $\left\{w_{i}\right\}_{i=1,2, \ldots}$ be a complete system of functions in $W_{0}^{1, p}(\Omega)$. We look for an approximate solution $u_{m}(x, t)$ in the form

$$
u_{m}(t)=\sum_{i=1}^{m} g_{i m}(t) w_{i}, g_{i m}(t) \in C^{1}([0, T])
$$

where the unknown functions $g_{i m}$ are determined by the following system of ordinary differential equations:

$$
\left(u_{m}^{\prime}(t), w_{j}\right)+a\left(u_{m}(t), w_{j}\right)=\left(\varphi\left(u_{m}(t)\right), w_{j}\right), 1 \leqq j \leqq m,{ }^{2)}
$$

with initial condition

$$
\begin{array}{r}
u_{m}(0)=u_{0 m}, u_{0 m}=\sum_{i=1}^{m} \alpha_{i m} w_{i} \longrightarrow u_{0} \text { in } W_{0}^{1, p}(\Omega), \\
\text { strongly as } m \rightarrow \infty
\end{array}
$$

Here

$$
a(u, v)=\sum_{i=1}^{n} \int_{\Omega}\left|\frac{\partial u}{\partial x_{i}}\right|^{p-2} \frac{\partial u}{\partial x_{i}} \frac{\partial v}{\partial x_{i}} d x
$$

Then we obtain the following a priori estimates:

$$
\begin{aligned}
& \left\|u_{m}\right\|_{L^{\infty}\left(0, T ; W_{0}^{1}, p(\Omega)\right)} \leqq c \\
& \left\|u_{m}^{\prime}\right\|_{L^{2}\left(0, T ; L^{2}(\Omega)\right)} \leqq c
\end{aligned}
$$

2) For the sake of simplicity, by the symbol' we denote the differentiation with respect to $t$. 
where $c$ is a constant independent of $\mathrm{m}^{3}$ )

Indeed, multiplying the $i$-th equation in (3.4) by $g_{i m}^{\prime}$, summing over $i$ from 1 to $m$ and integrating with respect to $t$, we get

$$
\begin{gathered}
\int_{0}^{t}\left\|u_{m}^{\prime}(s)\right\|_{L^{2}(\Omega)}^{2} d s+\frac{1}{p} \sum_{i=1}^{n}\left\|\frac{\partial}{\partial x_{i}} u_{m}(t)\right\|_{L^{p}(\Omega)}^{p}-\frac{1}{2+\alpha} \int_{\Omega} \Phi\left(u_{m}(x, t)\right) d x \\
=\frac{1}{p} \sum_{i=1}^{n}\left\|\frac{\partial}{\partial x_{i}} u_{0 m}\right\|_{L^{p}(\Omega)}^{p}-\frac{1}{2+\alpha} \int_{\Omega} \Phi\left(u_{0 m}(x)\right) d x
\end{gathered}
$$

Using Lemma 2 and Young's inequality, we have

$$
\begin{aligned}
\int_{0}^{t}\left\|u_{m}^{\prime}(s)\right\|_{L^{2}(\Omega)}^{2} d s+\frac{1}{p} \sum_{i=1}^{n}\left\|\frac{\partial}{\partial x_{i}} u_{m}(t)\right\|_{L^{p}(\Omega)}^{p} \\
\leqq c+\frac{1}{2+\alpha}\left\|u_{m}(t)\right\|_{L^{2+\alpha}(\Omega)}^{2+\alpha} \\
\leqq c+c\left(\sum_{i=1}^{n}\left\|\frac{\partial}{\partial x_{i}} u_{m}(t)\right\|_{L^{p}(\Omega)}^{p}\right)^{(2+\alpha) / p} \\
\leqq \frac{1}{2 p}\left(\sum_{i=1}^{n}\left\|\frac{\partial}{\partial x_{i}} u_{m}(t)\right\|_{L^{p}(\Omega)}^{p}\right)+c
\end{aligned}
$$

from which it follows that

$$
\int_{0}^{t}\left\|u_{m}^{\prime}(s)\right\|_{L^{2}(\Omega)}^{2} d s \leqq c
$$

and

$$
\sum_{i=1}^{n}\left\|\frac{\partial}{\partial x_{i}} u_{m}(t)\right\|_{L^{p}(\Omega)}^{p} \leqq c
$$

which imply (3.6) and (3.7).

From a priori estimates (3.6), (3.7) and Aubin's compactness theorem, ${ }^{4}$ ) we see that there exist a function $u$ and a subsequence $\left\{u_{\mu}\right\}$ of $\left\{u_{m}\right\}$ such that

$$
u_{\mu} \longrightarrow u \text { in } L^{\infty}\left(0, T ; W_{0}^{1, p}(\Omega)\right) \text { weakly star, }
$$

3) In the sequel of this note, $c$ denote various positive constants independent of $m$. 4) See $[7]$. 


$$
u_{\mu}^{\prime} \longrightarrow u^{\prime} \text { in } L^{2}\left(0, T ; L^{2}(\Omega)\right) \text { weakly, }
$$

$$
u_{\mu}(T) \longrightarrow u(T) \text { in } W_{0}^{1, p}(\Omega) \text { weakly, }
$$

$$
u_{\mu} \longrightarrow u \text { in } L^{2+\alpha}\left(0, T ; L^{2+\alpha}(\Omega)\right) \text { strongly }
$$

and

$$
A u_{\mu} \longrightarrow \chi \text { in } L^{\infty}\left(0, T ; W^{-1, p /(p-1)}(\Omega)\right)
$$

weakly star.

Then well known arguments of the theory of monotone operators yields

$$
\chi=A u
$$

which implies the function $u$ is a desired solution of the problem (1.1')(1.3).

Uniqueness part of Theorem 1 is easily proved as follows:

Let $u_{1}$ and $u_{2}$ be two solutions of the problem (1.1'), (1.2), (1.3) satisfying the same initial condition. Then the difference $w=u_{1}-u_{2}$ satisfies

$$
\begin{gathered}
w^{\prime}+A u_{1}-A u_{2}=\varphi\left(u_{1}\right)-\varphi\left(u_{2}\right)=\tilde{\varphi} w, \\
\tilde{\varphi}=\int_{0}^{1} \frac{d \varphi}{d u}\left(\theta u_{1}+(1-\theta) u_{2}\right) d \theta, \\
w(x, 0)=0 .
\end{gathered}
$$

Taking the scalar product of (3.15) with $w$ and using the monotonicity property of $A$ and Lemma 2, we have

$$
\begin{aligned}
\frac{1}{2} \frac{d}{d t}\|w\|_{L^{2}(\Omega)}^{2} & \leqq(\tilde{\varphi} w, w) \leqq \sup |\tilde{\varphi}|\|w\|_{L^{2}(\Omega)}^{2} \\
& \leqq \text { constant }\|w\|_{L^{2}(\Omega)}^{2}
\end{aligned}
$$

which implies

$$
w \equiv 0 .
$$

We now prove the last assertion of Theorem 1 . 
Let $V\left(0, T ; W_{0}^{1, p}(\Omega)\right)$ be the space of all the functions $v(t)$ such that $v(t) \in L^{2}\left(0, T ; W_{0}^{1, p}(\Omega)\right) \quad$ with $\quad v^{\prime}(t) \in L^{2}\left(0, T ; L^{2}(\Omega)\right)$.

Multiplying (3.4) by an arbitrary $C^{1}$-function $f(t)$ and integrating over $[0, t]$, we have

$$
\int_{0}^{t}\left(u_{m}^{\prime}(s), f(s) w_{j}\right) d s+\int_{0}^{t} a\left(u_{m}(s), f(s) w_{j}\right) d s=\int_{0}^{t}\left(\varphi\left(u_{m}(s)\right), f(s) w_{j}\right) d s .
$$

Taking the limit of both sides with $m=\mu, j$ fixed, we get

$$
\begin{gathered}
\int_{0}^{t}\left(u^{\prime}(s), f(s) w_{j}\right) d s+\int_{0}^{t} a\left(u(s), f(s) w_{j}\right) d s=\int_{0}^{t}\left(\varphi(u(s)), f(s) w_{j}\right) d s \\
\text { for } \quad{ }^{\forall}
\end{gathered}
$$

which implies

$$
\begin{gathered}
\int_{0}^{t}\left(u^{\prime}(s), \psi(s)\right) d s+\int_{0}^{t} a(u(s), \psi(s)) d s=\int_{0}^{t}(\varphi(u(s)), \psi(s)) d s \\
\text { for } \quad{ }^{\forall} \psi(s) \in V\left(0, T ; W_{0}^{1, p}(\Omega)\right) .
\end{gathered}
$$

In particular, setting $\psi(s)=v(s)-u(s)$ where $v(s)=\sup \{u(s), 0\},{ }^{5)}$ we have

$$
\begin{gathered}
\int_{0}^{t}\left(u^{\prime}(s), v(s)-u(s)\right) d s+\int_{0}^{t} a(u(s), v(s)-u(s)) d s \\
=\int_{0}^{t}(\varphi(u(s)), v(s)-u(s)) d s .
\end{gathered}
$$

From the definition of $v(s)$, we immediately get

$$
\begin{aligned}
& \int_{0}^{t}\left(v^{\prime}(s), v(s)-u(s)\right) d s=0, \\
& \int_{0}^{t} a(v(s), v(s)-u(s)) d s=0
\end{aligned}
$$

and

5) Note that $v(s) \in V\left(0, T ; W_{0}^{1, p}(\Omega)\right)$. 


$$
\int_{0}^{t}(\varphi(u(s)), v(s)-u(s)) d s=0
$$

Hence, from (3.18), we obtain

$$
\begin{aligned}
& \int_{0}^{t}\left(u^{\prime}(s)-v^{\prime}(s), v(s)-u(s)\right) d s \\
& \quad=\int_{0}^{t} a(v(s), v(s)-u(s)) d s-\int_{0}^{t} a(u(s), v(s)-u(s)) d s \\
& \quad \geqq 0
\end{aligned}
$$

which implies

$$
\|v(t)-u(t)\|_{L^{2}(\Omega)}^{2} \leqq\|v(0)-u(0)\|_{L^{2}(\Omega)}^{2}
$$

Hence we have

$$
u(x, t) \geqq 0 \quad \text { a.e. in } \Omega \text { and } t \geqq 0
$$

if $u_{0}(x) \geqq 0$ a.e. in $\Omega$.

Thus we have the theorem.

Remark 4. When $n<p$, in addition to the hypotheses of Theorem 1 , we suppose $A\left(u_{0}\right) \in L^{2}(\Omega)$. Then we have (see Lions [2])

$$
\partial u / \partial t \in L^{\infty}\left(0, T ; L^{2}(\Omega)\right) .
$$

Remark 5. When $p=2+\alpha$, so long as $C_{2}<1$, i.e., domain is sufficiently small, we easily see that the assertions of Theorem 1 also hold.

\section{Global Existence and Uniqueness When $p<2+a$}

Theorem 2. Suppose that $p<2+\alpha$ if $n \leqq p$ and $p<2+\alpha \leqq n p /$ $(n-p)$ if $n>p$. For every initial function $u_{0}(x)$ contained in the stable set $\mathscr{W}$ the initial-boundary value problem (1.1'), (1.2), (1.3) has a solution $u(x, t)$ contained in $\overline{\mathscr{W}}^{6)}$ such that

6) We denote by $\overline{\mathscr{W}}$ the closure of $\mathscr{W}$ in $W_{0}^{1, p}(\Omega)$. 


$$
u \in L^{\infty}\left(0, T ; W_{0}^{1, p}(\Omega)\right)
$$

and

$$
\partial u / \partial t \in L^{2}\left(0, T ; L^{2}(\Omega)\right)
$$

Furthermore we have $t>0$, hence $u(x, t)$

$$
\|u(t)\|_{L^{2}(\Omega)} \leqq\|u(s)\|_{L^{2}(\Omega)} \quad \text { if } \quad t \geqq s \geqq 0 \text {. }
$$

If $n<p$, the solution is uniquely determined by the initial function.

If $u_{0}(x) \geqq 0$ a.e. in $\Omega$, the solution $u(x, t) \geqq 0$ a.e. in $\Omega$ for any fixed $t>0$, hence $u(x, t)$ is a solution of the problem (1.1)-(1.3).

Proof. The Galerkin's method is again employed. Let $\left\{w_{i}\right\}_{i=1,2} \ldots$ and an approximate solution $u_{m}$ be the same as those stated in the proof of Theorem 1. Let $\left\{u_{0 m}\right\}$ be a sequence such that

$$
\begin{array}{r}
u_{0 m} \in \mathscr{W}, u_{0 m}=\sum_{i=1}^{m} \alpha_{i m} w_{i} \longrightarrow u_{0} \text { in } W_{0}^{1, p}(\Omega) \\
\text { strongly as } m \longrightarrow \infty .
\end{array}
$$

We have local existence of $u_{m}(t)$ i.e. in $\left[0, t_{m}\right], t_{m}>0$ and in this interval (c.f. (3.8)):

$$
\int_{0}^{t}\left\|u_{m}^{\prime}(s)\right\|_{L^{2}(\Omega)}^{2} d s+J\left(u_{m}(t)\right)=J\left(u_{0 m}\right)
$$

We will show that

$$
u_{m}(t) \in \mathscr{W}, \quad{ }^{\forall} t \geqq 0
$$

Suppose that (4.6) does not hold and let $t^{*}$ be the smallest time for which $u_{m}\left(t^{*}\right) \notin \mathscr{W}$. Then in virtue of the continuity of $u_{m}(t)$ we see that $u_{m}\left(t^{*}\right) \in \partial \mathscr{W}$. Hence, from Lemma 4 we have

$$
J\left(u_{m}\left(t^{*}\right)\right)=d
$$

or

$$
a\left(u_{m}\left(t^{*}\right)\right)-b\left(u_{m}\left(t^{*}\right)\right)=0
$$


which contradicts the equality (4.5) and the fact that the initial function $u_{0 m}$ is contained in $\mathscr{W}$. Indeed, when (4.7) holds, the assertion is obvious and when (4.8) holds, we have

$$
J\left(u_{m}\left(t^{*}\right)\right)=J\left(\left(\frac{a\left(u_{m}\left(t^{*}\right)\right)}{b\left(u_{m}\left(t^{*}\right)\right)}\right)^{1 /(2+\alpha-p)} u_{m}\left(t^{*}\right)\right) \geqq d
$$

which also implies the contradiction.

Then from the equality (4.5) and Lemma 4, we get

$$
\int_{0}^{t}\left\|u_{m}^{\prime}(s)\right\|_{L^{2}(\Omega)}^{2} d s+\left(\frac{1}{p}-\frac{1}{2+\alpha}\right) a\left(u_{m}(t)\right) \leqq J\left(u_{0 m}\right) \leqq c
$$

which implies

$$
\left\|u_{m}\right\|_{L^{\infty}\left(0, T ; W_{0}^{1, p}(\Omega)\right)} \leqq c
$$

and

$$
\left\|u_{m}^{\prime}\right\|_{L^{2}\left(0, T ; L^{2}(\Omega)\right)} \leqq c .
$$

From Aubin's compactness theorem and well known arguments of the theory of monotone operators, we see that there exist a function $u$ and a subsequence $\left\{u_{\mu}\right\}$ of $\left\{u_{m}\right\}$ such that (3.9)-(3.13) are fulfilled and $u$ is a solution of the equation (1.1') with conditions (1.2), (1.3).

We now prove (4.3).

Since $u$ is contained in $\overline{\mathscr{W}}$ from Lemma 4 , we see that

$$
a(u(t))-b(u(t)) \geqq 0 .
$$

On the other hand, setting $\psi(s)=u(s)$ in (3.17) with some modifications, we easily obtain

$$
\frac{1}{2}\|u(t)\|_{L^{2}(\Omega)}^{2}=\frac{1}{2}\|u(s)\|_{L^{2}(\Omega)}^{2}-\int_{s}^{t} a(u(\tau)) d \tau+\int_{s}^{t} b(u(\tau)) d \tau
$$

Hence, the inequality (4.3) immediately follows from (4.12) and (4.13).

Proofs of the last two assertions of Theorem 2 are easily obtained by a repetition of the arguments in the proof of Theorem 1.

Remark 6. When $n<p$, in addition to the hypotheses of Theorem 2, 
we assume that $A\left(u_{0}\right) \in L^{2}(\Omega)$. Then we have

$$
\partial u / \partial t \in L^{\infty}\left(0, T ; L^{2}(\Omega)\right) .
$$

\section{Nonexistence of Global Solutions}

Theorem 3. (local existence) Suppose that $p<2+\alpha<2 p / n+p$ and $u_{0}(x) \in W_{0}^{1, p}(\Omega)$. Then there exists a positive constant $T_{0}$ such that in the interval $0 \leqq t \leqq T_{0}$ the problem (1.1'), (1.2), (1.3) has a solution $u(x, t)$ such that

$$
\begin{aligned}
& u \in L^{\infty}\left(0, T_{0} ; W_{0}^{1, p}(\Omega)\right) \\
& \partial u / \partial t \in L^{2}\left(0, T_{0} ; L^{2}(\Omega)\right)
\end{aligned}
$$

and satisfying

$$
\frac{1}{2}\|u(t)\|_{L^{2}(\Omega)}^{2}+\int_{0}^{t} a(u(s)) d s-\int_{0}^{t} b(u(s)) d s=\frac{1}{2}\left\|u_{0}\right\|_{L^{2}(\Omega)}^{2}
$$

and

$$
J(u(t)) \leqq J\left(u_{0}\right), \quad \text { a.a. } \quad t \in\left[0, T_{0}\right]
$$

If $u_{0}(x) \geqq 0$ a.e. in $\Omega, u(x, t) \geqq 0$ a.e. in $\Omega$ for any fixed $t \in\left[0, T_{0}\right]$ and $u(x, t)$ is a solution of problem (1.1)-(1.3).

Theorem 4. Suppose that all the conditions of Theorem 3 are fulfilled. Furthemore we assume that $u_{0}(x) \geqq 0$ a.e. in $\Omega$,

$$
J\left(u_{0}(x)\right)<0
$$

and

$$
\left\|u_{0}\right\|_{L^{2}(\Omega)} \geqq(\operatorname{mes}(\Omega))^{1 / 2} \frac{(2+\alpha)^{1 / \alpha}}{\alpha^{1 / \alpha}(2+\alpha-p)^{1 / \alpha} T^{1 / \alpha}}
$$

Then the local solution of the problem (1.1)-(1.3) corresponding to this initial function is not continued globally, i.e., blows up in a finite time $<T$.

Proof of Theorem 3. The Galerkin's method is employed. Let 
$\left\{w_{i}\right\}_{i=1,2}, \cdots,\left\{u_{m}\right\}$ and $\left\{u_{0 m}\right\}$ be the same as those stated in the proof of Theorem 1 .

Multiplication of the $i$-th equation in (3.4) by $g_{i m}$ and summation over $i$ from 1 to $m$ give

$$
\frac{1}{2} \frac{d}{d t}\left\|u_{m}\right\|_{L^{2}(\Omega)}^{2}+\sum_{i=1}^{n}\left\|\frac{\partial}{\partial x_{i}} u_{m}(t)\right\|_{L^{p}(\Omega)}^{p}=\int_{\Omega} \Phi\left(u_{m}(x, t)\right) d x .
$$

In virtue of Lemma 1 we have

$$
\left|\int_{\Omega} \Phi\left(u_{m}(x, t)\right) d x\right| \leqq C_{1}\left(\sum_{i=1}^{n}\left\|\frac{\partial}{\partial x_{i}} u_{m}\right\|_{L^{p}(\Omega)}^{p}\right)^{\delta}\left\|u_{m}\right\|_{L^{2}(\Omega)}^{\delta^{\prime}}
$$

where

$$
\delta=\frac{\alpha n}{2 p-2 n+n p}<1
$$

and

$$
\delta^{\prime}=\frac{(\alpha+2)(2 p-2 n+n p)-\alpha n p}{2 p-2 n+n p}>0 .
$$

Young's inequality gives

$$
\left|\int_{\Omega} \Phi\left(u_{m}(x, t)\right) d x\right| \leqq \frac{1}{2 p}\left(\sum_{i=1}^{n}\left\|\frac{\partial}{\partial x_{i}} u_{m}\right\|_{L^{p}(\Omega)}^{p}\right)+\frac{c}{2}\left\|u_{m}\right\|_{L^{2}(\Omega)}^{\delta^{\prime \prime}},
$$

where $\delta^{\prime \prime}=\delta^{\prime} / 2(1-\delta)>1$.

Hence, from (5.5) and (5.7) we get

$$
\frac{d}{d t}\left\|u_{m}\right\|_{L^{2}(\Omega)}^{2} \leqq c\left\|u_{m}\right\|_{L^{2}(\Omega)}^{\delta^{\prime \prime}}
$$

It follows immediately that the solution $\left\|u_{m}\right\|_{L^{2}(\Omega)}^{2}$ of this differential inequality can be majorized by the solution of the initial value problem;

$$
\frac{d y}{d t}=c y^{\delta^{\prime \prime}}, \quad y(0)=y_{0} \geqq\left\|u_{0 m}\right\|_{L^{2}(\Omega)}^{2}
$$

The solution $y$ of (5.9) is finite only if

$$
t<t_{\infty}=1 /\left(c\left(\delta^{\prime \prime}-1\right) y_{0}^{\delta^{\prime \prime}-1}\right) .
$$


Hence, in the interval $0 \leqq t \leqq T_{0}=t_{\infty} / 2$, we have an estimate

$$
\left\|u_{m}(t)\right\|_{L^{2}(\Omega)}^{2} \leqq c .
$$

From (3.8), (5.7) and (5.11) we immediately get

$$
\left\|u_{m}\right\|_{L^{\infty}\left(0, T_{0} ; W_{0}^{1, p}(\Omega)\right)} \leqq c
$$

and

$$
\left\|u_{m}^{\prime}\right\|_{L^{2}\left(0, T_{0} ; L^{2}(\Omega)\right)} \leqq c
$$

from which, by a repetition of the arguments in the proof of Theorem 1 , we see that the problem $\left(1.1^{\prime}\right)-(1.3)$ has a solution $u$ in the interval $0 \leqq t \leqq T_{0}$.

We now show that the solution $u$ satisfies (5.1) and (5.2).

Setting $\psi(s)=u(s)$ in (3.17) we immediately have the equality (5.1).

From (3.8) we get

$$
J\left(u_{m}(t)\right) \leqq J\left(u_{0 m}\right)
$$

Let $\theta$ be the function which lies in $C\left(\left[0, T_{0}\right]\right)$ and is nonnegative. Then from (5.14) with $m=\mu$ we get

$$
\int_{0}^{T_{0}} J\left(u_{\mu}(t)\right) \theta(t) d t \leqq \int_{0}^{T_{0}} J\left(u_{0 \mu}\right) \theta(t) d t
$$

The second member tends to

$$
\int_{0}^{T_{0}} J\left(u_{0}\right) \theta(t) d t
$$

as $\mu \rightarrow \infty$.

The first member is lower semi-continuous with respect to the weak topology of $L^{2}\left(0, T ; W_{0}^{1, p}(\Omega)\right)$.

Hence

$$
\int_{0}^{T_{0}} J(u(t)) \theta(t) d t \leqq \liminf _{\mu \rightarrow \infty} \int_{0}^{T_{0}} J\left(u_{\mu}(t)\right) \theta(t) d t \leqq \int_{0}^{T_{0}} J\left(u_{0}\right) \theta(t) d t
$$

Since $\theta(t)$ is arbitrary, we obtain 


$$
J(u(t)) \leqq J\left(u_{0}\right), \quad \text { a.a. } t \in\left[0, T_{0}\right] .
$$

This completes the proof of Theorem 3 .

Proof of Theorem 4. Note that if $u_{0}(x) \geq 0$ a.e. in $\Omega$, the solution $u(x, t) \geqq 0$ a.e. in $\Omega$ for any $t \geqq 0$.

Suppose that the assertion of Theorem 4 does not hold and let $u(x, t)$ be the global solution corresponding to the initial function $u_{0}(x)$ satisfying the assumptions stated in this Theorem. Then $u(x, t)$ satisfies (5.1) and (5.2) for ${ }^{\forall} t>0$.

Then from (5.2) and (5.3) we have

$$
\frac{1}{p} a(u(t))-\frac{1}{2+\alpha} b(u(t))<0, \quad \text { a.a. } t \geqq 0 .
$$

Substituting (5.16) into (5.1) we get

$$
\begin{aligned}
\frac{1}{2}\|u(t)\|_{L^{2}(\Omega)}^{2} \geqq & \left(1-\frac{p}{2+\alpha}\right) \int_{0}^{t} b(u(s)) d s+\frac{1}{2}\left\|u_{0}\right\|_{L^{2}(\Omega)}^{2} \\
\geqq & \frac{2+\alpha-p}{2+\alpha}(\operatorname{mes}(\Omega))^{-\alpha / 2} \int_{0}^{t}\|u(s)\|_{L^{2}(\Omega)}^{2+\alpha} d s \\
& +\frac{1}{2}\left\|u_{0}\right\|_{L^{2}(\Omega)}^{2} .
\end{aligned}
$$

Here we have used the inequality

$$
\left.\left\|\left.u\right|_{L^{2}(\Omega)} ^{2} \leqq(\operatorname{mes}(\Omega))^{\alpha / 2+\alpha}\right\| u\right|_{L^{2+\alpha}(\Omega)} ^{2} .
$$

From (5.17) we immediately obtain

$$
\|u(t)\|_{L^{2}(\Omega)}^{\alpha} \geqq \frac{1}{\left\|u_{0}\right\|_{L^{2}(\Omega)}^{-\alpha}-L t}
$$

where

$$
L=\frac{\alpha(2+\alpha-p)}{2+\alpha}(\operatorname{mes}(\Omega))^{-\alpha / 2} .
$$

Hence $\|u(t)\|_{L^{2}(\Omega)}$ diverges to $+\infty$ as $t \rightarrow \frac{1}{L}\left\|u_{0}\right\|_{L^{2}(\Omega)}^{-\alpha}<T$. This contradicts that $u$ is a global solution. Thus we have the theorem. 
Remark 7. When $p=2$, local existence theorem of the classical solutions of the problem (1.1)-(1.3) is of course established without any restrictions upon the growth order $\alpha$ (see Friedman [5]). Theorem 4 gives another approach to "blowing up" of the solution of the equation (1.4) with conditions (1.2), (1.3).

Remark 8. If we add the term $\Delta u$ to the second members of the equation (1.1), for smooth initial data, the problem (1.1)-(1.3) has a classical unique solution which may local in $t$, without any restrictions upon the growth orders $p$ and $\alpha$ (see Sobolevskii [6]). Then by the analogous method to that stated in Theorem 4, we can prove the solution blowing up in a finite time if the initial condition satisfies the assumptions stated in Theorem 4.

\section{Final Remarks}

In this section, as compared with the equation (1.1), we consider the following equation

$$
\frac{\partial u}{\partial t}=\sum_{i=1}^{n} \frac{\partial}{\partial x_{i}}\left(\left|\frac{\partial u}{\partial x_{i}}\right|^{p-2} \frac{\partial u}{\partial x_{i}}\right)-u^{1+\alpha}, t>0, x \in \Omega
$$

with conditions (1.2) and (1.3).

Then by the analogous methods to those stated in $\S 3$, we can easily obtain :

Theorem 5. Let $2 \leqq p<\infty$ and $\alpha \geqq 0$. Suppose that $u_{0}(x) \in$ $W_{0}^{1, p}(\Omega) \cap L^{2+\alpha}(\Omega)$ and $u_{0}(x) \geqq 0$ a.e. in $\Omega$. Then there exists one and only one function $u$ such that

$$
\begin{gathered}
u(x, t) \geqq 0 \text { a.e. in } \Omega \text { for any fixed } t>0, \\
u \in L^{\infty}\left(0, T ; W_{0}^{1, p}(\Omega) \cap L^{2+\alpha}(\Omega)\right), \\
\partial u / \partial t \in L^{2}\left(0, T ; L^{2}(\Omega)\right)
\end{gathered}
$$

and which satisfies (6.1) in a generalized sense. 
Furthermore, if $A\left(u_{0}\right) \in L^{2}(\Omega)$, then the solution $u$ satisfies

$$
\partial u / \partial t \in L^{\infty}\left(0, T ; L^{2}(\Omega)\right)
$$

Remark 9. The assertions of Theorem 5 are also valid if we replace the term $u^{1+\alpha}$ by more general nonlinear term $f(u)$ satisfying $f(u)>0$ (for $u>0$ ) and $f(0)=0$.

Remark 10. When $\alpha$ is an odd integer, if we put off the positivity property of $u_{0}$, we must impose the condition that $u_{0}$ is contained in the stable set $W$ analogously defined to the $\S 2$ in order to obtain global existence theorems.

\section{Acknowledgements}

The author wishes to express his sincere gratitude to Professor $\mathrm{R}$. Iino for his kind advices and his constant encouragement.

\section{References}

[1] Fujita, H., On some nonexistence and nonuniqueness theorems for nonlinear parabolic equations. Proc. Symp. in Pure Math. 18, American Mathematical Society, Providence, Rhode Island, (1970), 105-113.

[2] Lions, J.-L., Quelques Méthodes de Résolution des Problèmes aux Limites non Linéaires, Dunod, Paris, 1969.

[3] Ladyzhenskaya, O.A., V.A. Solonnikov and N. N. Uralceva, Linear and Quasilinear Parabolic Eqnations, "Nauka", Moscow, 1967; English transl., Trans. Math. Monographs, 23, American Mathematical Society, Providence, Rhode Island, 1968.

[4] Sattinger, D.H., On global solution of nonlinear hyperbolic equations, Arch. Rat. Mech. Anal. 30 (1968), 148-172.

[5] Friedman, A., Partial Differential Equations of Parabolic Type, Prentice-Hall, Englewood Cliffs, N. J., 1964.

[6] Sobolevskii, P.E. Local and nonlocal existence theorems for nonlinear secondorder parabolic equations, Dokl. Akad. Nauk SSSR, 136 (1961), 292-295.

[7] Aubin, J.P., Un théorème de compacité, C. R. Acad. Sci. Paris, 256 (1963), 5042 5044. 
